\title{
Pellet Size Estimation Using Spherical Fitting
}

\author{
T. Andersson, M. J. Thurley, O. Marklund \\ EISLAB, \\ Department of Computer Science and Electrical Engineering, \\ Luleå University of Technology, 97187 Luleå \\ E-mail: \{tobias.andersson, matthew.thurley, olov.marklund $\} @ 1 t u . s e$ \\ URL: www.csee.ltu.se/ $\sim$ tobiasa, www.csee. 1 tu.se $/ \sim \mathrm{mjt}$
}

\begin{abstract}
Evaluation of Spherical Fitting as a technique for sizing iron ore pellets is performed. Size measurement of pellet in industry is usually performed by manual sampling and sieving techniques. Automatic on-line analysis of pellet size would allow non-invasive, frequent and consistent measurement. Previous work has used an assumption that pellets are spherical to estimate pellet sizes. In this research we use a $3 D$ laser camera system in a laboratory environment to capture 3D surface data of pellets and steel balls. Validation of the $3 D$ data against a spherical model has been performed and demonstrates that pellets are not spherical and have physical structures that a spherical model cannot capture.
\end{abstract}

Keywords - Size estimation, Spherical fitting, Model evaluation, Industrial monitoring, Material analysis, Image analysis.

\section{INTRODUCTION}

Pellet's sizes are critical to the efficiency of the blast furnace process in production of steel. Ökvist et al. [1] shows how different pellet size distributions effect the blast furnace process. Overly coarse pellets effect the blast furnace process negatively and Ökvist et al. [1] reports on how to minimize the effect by operating the furnace with different parameters. An on-line system for measurement of pellet sizes would improve productivity through fast feedback and efficient control of the blast furnace.

In pellet manufacturing, manual sampling followed by sieving with a square mesh is used for quality control. The manual sampling is performed infrequently and is time-consuming. Fast feedback of pellets sizes is desirable.

Blomquist and Wernerson [2] use a statistical model that assumes pellets are spherical to measure diameter and diameter deviation of pellets from chord lengths.

Bouajila et al. [3] estimate size of green pellets with a 3D laser camera. Based on the assumption that pellets are spherical, they apply a spherical smoothing method to obtain a complete size distribution of the produced pellets. Bouajila et al. [3] report that the estimated size distribution correlates well with the reference values.

In the presented research we use an industrial prototype 3D imaging system to capture 3D surface data of pellet piles on a conveyor. Estimation of pellet sizes is made based on the assumption that pellets are spherical. An evaluation of the suitability of the spherical assumption is made.

\section{METHODS}

In this section we outline the methods used to capture the 3D surface data, segment it, determine segmented regions sizes and evaluate the spherical model.

\section{A. Imaging System}

An imaging system that captures 3D surface data has been implemented by MBV Systems [4]. The system is based on a projected laser line and camera triangulation [5, triangulation, structured light]. It has a continuous wave diode laser with line generating optics and a high speed digital camera capable of 4000 frames per second. The angle between the line of sight and the laser is approximately 30 degrees.

\section{B. Collection of Data}

B.1 Pellets

Mechanical sieving is the accepted industry technique for sizing pellets. A sample of baked pellets was sieved into 6 size gradings and is shown in table $\mathrm{I}$.

\begin{tabular}{l}
\begin{tabular}{|l|c|c|c|}
\hline Sieve size $(\mathrm{mm}) \dagger$ & Weight $(\mathrm{kg})$ & $\%$ & Cum. \% \\
\hline 6.3 & 4.089 & 12.9 & 12.9 \\
9 & 4.755 & 14.9 & 27.8 \\
10 & 12.613 & 39.6 & 67.4 \\
11.2 & 7.493 & 23.5 & 91.0 \\
12.5 & 2.445 & 7.68 & 98.7 \\
14 & 0.4233 & 1.33 & 100 \\
\hline
\end{tabular} \\
\hline \multicolumn{3}{|c|}{ The lower bound of each sieve size increment } \\
TABLE I. Sieve size distribution of the sample of baked pellets.
\end{tabular}


Each size class is captured individually with the imaging system in a laboratory setup. The pellets are spread out on a conveyor belt to make sure that the surface of each pellet is not occluded by other pellets.

\section{B.2 Steel Balls}

The steel balls have a known diameter and have been painted with a thin layer of mat grey paint to allow data capture with the imaging system. The steel balls are positioned separately on a tray. The imaging system captures a sample of 45 balls each of size $5,10,12.7$ and $16 \mathrm{~mm}, 30$ balls of $17.5 \mathrm{~mm}$ and 15 balls each of 20 and $25 \mathrm{~mm}$.

\section{Segmentation Algorithm}

Pellet segmentation has been performed by Thurley [6] using a mathematical morpholgy implementation [7] for sparse, irregularly spaced 3D surface data.

This technique [6] applies a variety of algorithms including morphological and linear edge detection, distance transform, local maxima detection and watershed segmentation.

\section{Estimation of Size}

Using the segmented data each pellet is identified and its size may be estimated. To evaluate the assumption that pellets are spherical, pellet size is estimated by fitting a spherical model to the segmented data of each pellet.

To do this consider the equation of a sphere, which can be written as seen in equation 1 where $x_{0}, y_{0}$ and $z_{0}$ are the coordinates for the center of the sphere and $R$ is the radius of the sphere.

$f\left(x_{0}, y_{0}, z_{0}, R\right)=\left(x-x_{0}\right)^{2}+\left(y-y_{0}\right)^{2}+\left(z-z_{0}\right)^{2}-R^{2}=0$

By using the partial derivatives as seen in equation 2 it is possible to construct a linear least-squares problem with $m$ coordinate points $\left(x_{y}, y_{1}, z_{1}\right),\left(x_{2}, y_{2}, z_{1}\right), \ldots,\left(x_{m}, y_{m}, z_{m}\right)$.

$$
\frac{\partial f}{\partial x_{o}}=0, \frac{\partial f}{\partial y_{o}}=0, \frac{\partial f}{\partial z_{o}}=0, \frac{\partial f}{\partial R}=0
$$

The linear system $\mathbf{M a}=\mathbf{v}$ is shown in equation 3 where $\mathbf{M}$ is a $m$-by- 4 matrix and $\mathbf{v}$ is a $m$ long column vector.

$$
\left[\begin{array}{cccc}
x_{1} & y_{1} & z_{1} & 1 \\
x_{2} & y_{2} & z_{2} & 1 \\
\vdots & \vdots & \vdots & \vdots \\
x_{m} & y_{m} & z_{m} & 1
\end{array}\right]\left[\begin{array}{c}
a \\
b \\
c \\
d
\end{array}\right]=\left[\begin{array}{c}
-x_{1}^{2}-y_{1}^{2}-z_{1}^{2} \\
-x_{2}^{2}-y_{2}^{2}-z_{2}^{2} \\
\vdots \\
-x_{m}^{2}-y_{m}^{2}-z_{m}^{2}
\end{array}\right]
$$

The solution is given by $\mathbf{a}=\left(\mathbf{M}^{t} \mathbf{M}\right)^{-1}\left(\mathbf{M}^{t} \mathbf{v}\right)$ where vector $\mathbf{a}$ is given by equation 4 from which $x_{0}, y_{0}, z_{0}$ and $R$ can be determined.

$$
\left[\begin{array}{l}
a \\
b \\
c \\
d
\end{array}\right]=\left[\begin{array}{c}
-2 x_{0} \\
-2 y_{0} \\
-2 z_{0} \\
x_{0}^{2}+y_{0}^{2}+z_{0}^{2}-R^{2}
\end{array}\right]
$$

\section{E. Model Evaluation}

To validate the model we compare estimated values with measured values of steel ball's and pellet's size. The estimated values should clearly correlate with measured physical parameters if the spherical model is valid.

Residuals, which is the part of the data that the model could not reproduce, may also indicate how well the model describes the data. A spherical model's residuals are calculated by equation 5 , where $x_{i}, y_{i}$ and $z_{i}$ is the coordinates for point $i$.

$$
\epsilon_{i}=\sqrt{\left(x_{i}-x_{0}\right)^{2}+\left(y_{i}-y_{0}\right)^{2}+\left(z_{i}-z_{0}\right)^{2}}-R
$$

Ljung [8] suggest analysis of basic statistics for residuals and we will use root-mean-square error as shown in equation 6 in this analysis where $\epsilon_{i}$ is the residual for point $i$ and $m$ is the number of points.

$$
\text { RMS Error }=\sqrt{\frac{1}{m} \sum_{i=1}^{m} \epsilon_{i}^{2}}
$$

The comparison of estimated values with known data and the residual analysis give a good indication of how well the model captures the data.

\section{PERFORMANCE OF SPHERICAL FITTING}

The accuracy of the imaging system and the spherical model as a measurement method is evaluated by sizing perfect steel balls and sieved pellets.

\section{A. Steel Balls}

The model is fitted to each steel ball and the result of estimated sizes and residual statistics is shown in table II.

The median of the estimated sizes is close to the known values of the steel balls diameter. The residual analysis indicate some deviation between data and the model. The median of RMS Error range from 0.107 to 0.128 for all size classes.

For perfect steel balls the model and measurement system seems to give good results. The physical comparison to known sizes of the balls is very good. The residual analysis indicate that there is some deviation from the model but it is small. 


\begin{tabular}{|l|c|cc|cc|}
\hline $\begin{array}{l}\text { Size } \\
(\mathrm{mm})\end{array}$ & Nbr & \multicolumn{2}{|c|}{ Size est. ${ }^{*}(\mathrm{~mm})$} & \multicolumn{2}{c|}{ RMS Error $^{\dagger}$} \\
\hline 5 & 45 & 5.432 & 0.308 & 0.107 & 0.0133 \\
10 & 45 & 10.35 & 0.165 & 0.107 & 0.0073 \\
12.7 & 45 & 12.95 & 0.108 & 0.106 & 0.0048 \\
16 & 45 & 16.18 & 0.106 & 0.119 & 0.0054 \\
17.5 & 30 & 17.63 & 0.081 & 0.117 & 0.0059 \\
20 & 15 & 20.03 & 0.073 & 0.118 & 0.0059 \\
25 & 15 & 24.95 & 0.169 & 0.128 & 0.0099 \\
\hline
\end{tabular}

* Known diameter of steel ball

* Estimated size. Diameter of fitted sphere

$\dagger$ Root-mean-square error. Equation 6

$\diamond$ Interquartile range. Range between $25^{t h}$ and $75^{t h}$ percentile

TABLE II. Result for spherical fit to measured steel balls. Estimated sizes correlate well with known sizes and the RMS Error estimate is small. The interquartile range is small for both estimated diameter and RMS Error.

\begin{tabular}{|l|c|cc|cc|}
\hline $\begin{array}{l}\text { Size }^{*} \\
(\mathrm{~mm})\end{array}$ & Nbr & \multicolumn{2}{|c|}{ Size est. Median $^{\star}$} & IQR $^{\diamond}$ & \multicolumn{2}{c|}{ RMS Error $\dagger$} \\
\hline 6.3 & 1010 & 10.34 & 1.777 & 0.261 & IQR $^{\diamond}$ \\
\hline & 755 & 11.34 & 1.553 & 0.266 & 0.134 \\
10 & 867 & 12.12 & 1.504 & 0.274 & 0.110 \\
11.2 & 677 & 13.58 & 1.794 & 0.299 & 0.124 \\
12.5 & 477 & 15.02 & 1.887 & 0.329 & 0.133 \\
14 & 61 & 16.00 & 1.690 & 0.350 & 0.127 \\
\hline
\end{tabular}

* The lower bound of each sieve size increment

* Estimated size. Diameter of fitted sphere

$\dagger$ Root-mean-square error. Equation 6

$\diamond$ Interquartile range. Range between $25^{\text {th }}$ and $75^{\text {th }}$ percentile

TABLE III. Result for spherical fit to measured pellets. The estimated size is constantly overestimated and the interquartile range is relatively large. The RMS Error is large.

\section{B. Pellets}

To evaluate if a spherical assumption of pellets shape is adequate the spherical model is fitted to each pellet. The estimated diameters and statistics for the residuals for the different classes are calculated. We present the distribution of the estimated diameters and RMS error for the different classes in Table III. The result is also shown graphically in figures 1 and 2 using the graphical convention of horizontal box-plots.

The central portion of a box-plot contains a rectangular box. In the center of this box is a short thick vertical black line, this marks the median value (or 50th percentile) of the data. The left edge of the rectangular box marks the 25 th percentile, and the right edge marks the 75 th percentile. The difference be- tween the 75th and 25th percentile is the interquartile range (IQR). The IQR is a robust estimate of the spread of the data. The circular dots to the left and right of each box-plot indicate values that are statistically determined to be outliers. Values are defined to be outliers when they are less than the 25th percentile $-1.5 \mathrm{IQR}$ or greater than the 75 th percentile $+1.5 \mathrm{IQR}$. These values correspond to pellets that are particularly nonspherical. The dashed lines extending to the left and right of the rectangular box extend to the statistically valid min and max. The graphs and determination of outliers were calculated using the R statistical analysis package [9].

In figure 1 , it is clear that the size estimate for pellets is constantly overestimated. Also, it is important to notice that the interquartile range of the size estimates is generally larger than the intervals between the different classes. The classes are not clearly separable and will not be suitable for determining pellet size that corresponds to square mesh sieving techniques.

The median value of the RMS Error, shown in table III, range from 0.261 for the smallest size class to 0.366 for the biggest size class. The interquartile range is above 0.1 for all size classes. The RMS Error clearly indicates that pellets are not spherical and the distribution of the RMS error for the different classes can be seen in figure 2 .

The physical comparison to known sizes of pellets combined with the residual analysis indicate that pellets are not spherical. It is important to notice that the physical comparison of the calculated size and the known sieve sizes show that the estimated sizes are wrong and sensitive to input data.

For comparison we show the box-plots for steel balls in figures 3 and 4 drawn at the same scale as figure 1 and 2. Analyzing figure 3 and 1 , it is clear that the distributions for the estimated sizes are very narrow and close to the known sizes for steel balls. For pellets the distributions are broad and the size estimates are constantly overestimated. Analyzing figure 4 and 2, it is clear that the RMS Errors are comparatively large for pellets.

In addition, the residuals are shown in figure 5 for pellets in size class $10 \mathrm{~mm}$. For comparison we show the residuals for steel balls with a diameter of $10 \mathrm{~mm}$ in figure 6 . It is obvious that the model does not capture certain areas of pellets that deviate from a spherical model. The figures show a significantly greater variation of the residuals for pellets than for steel balls and this indicate that the spherical model works well for perfect steel balls but do not account for all variations in pellet's structure. 
Sphere Fitting Diameter for Pellets of Various Sizes

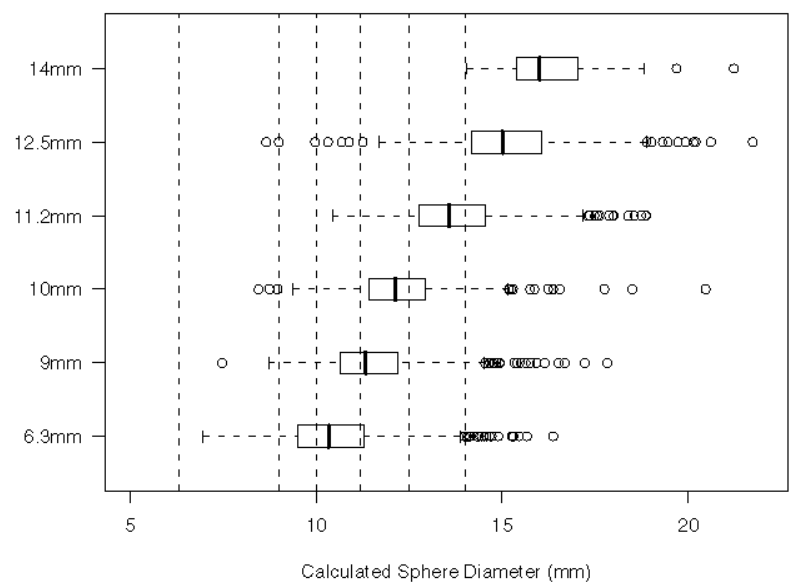

Fig. 1. Distribution of estimated sphere diameters for different size classes. The dashed lines corresponds to the lower bound of each size class.

Sphere Fitting RMS Error for Pellets of Various Sizes

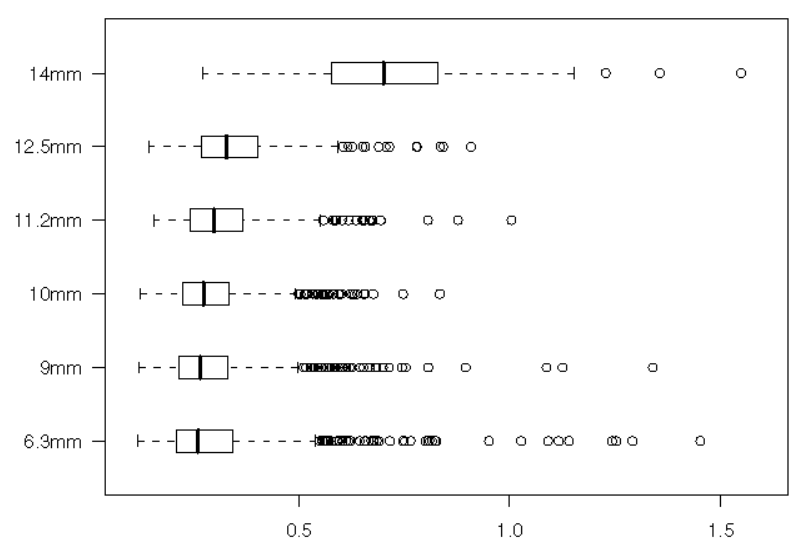

Fig. 2. Distribution of estimated error of fit for different size classes.
Sphere Fitting Diameter for Steel Balls of Various Diameters

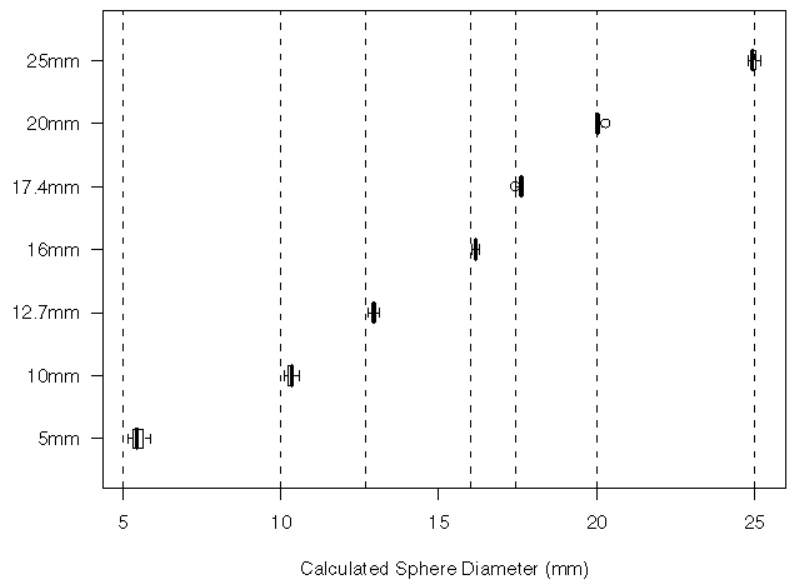

Fig. 3. Distribution of estimated sphere diameters for different size classes. The dashed lines corresponds to known diameters for the balls.
Sphere Fitting RMS Error for Steel Balls of Various Diameter

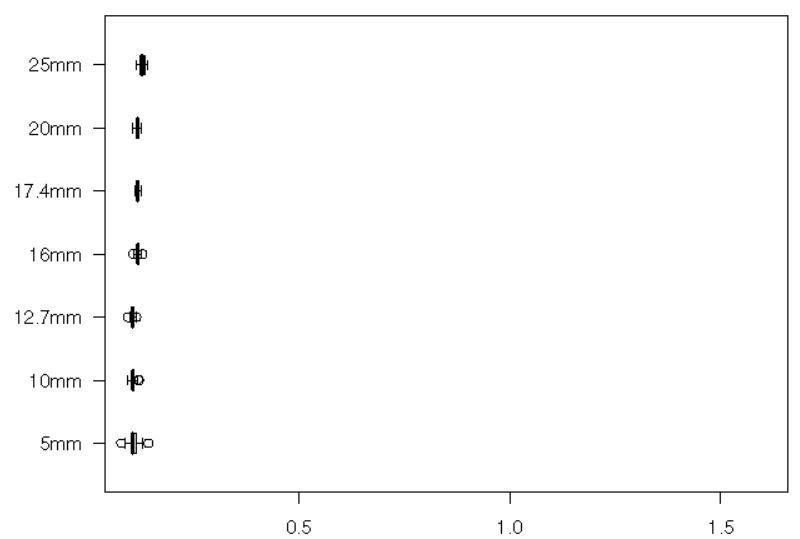

Fig. 4. Distribution of estimated error of fit for different size classes. 


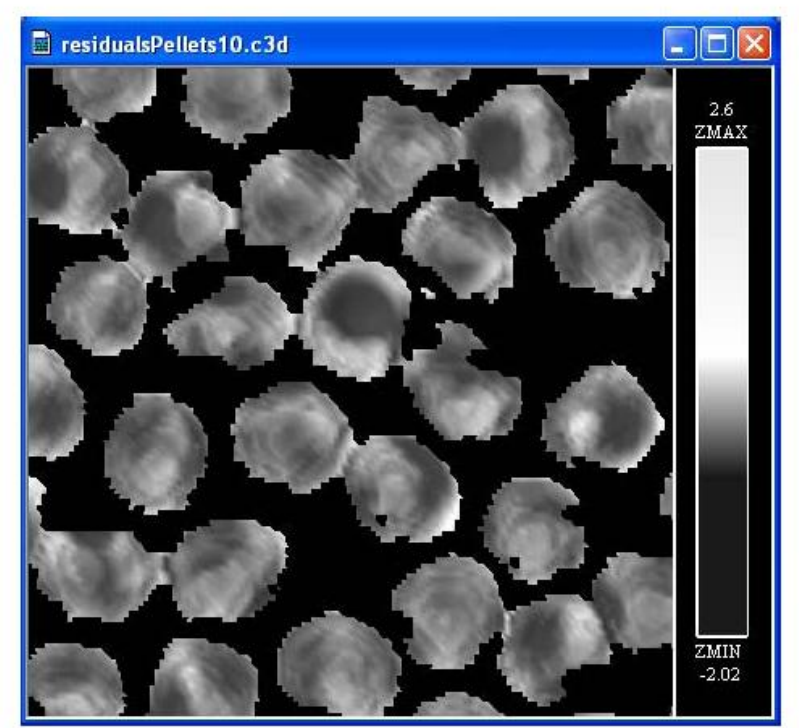

Fig. 5. Sample of residuals for pellets in class $10 \mathrm{~mm}$. That is pellets of size between 10 and $11.2 \mathrm{~mm}$.

\section{CONCLUSIONS}

A study of the adequacy of the assumption that pellets are spherical is made. Pellets are collected and mechanically sieved into different classes. Also perfect steel balls with well known properties are collected. The two samples are captured by an imaging system that produces 3D surface data. The surface is segmented and a spherical model fitted to each pellet and steel ball. Model evaluation based on physical properties and residual analysis show that the spherical model works well for perfect steel balls but not for pellets.

\section{ACKNOWLEDGMENT}

We wish to thank the staff at ProcessIT Innovations for all time and effort to make this research possible. We thank John Erik Larsson at MBV-systems for adjustments and development of the 3D surface imaging system. We thank Kjell-Ove Mickelsson and Robert Johannson for their invaluable industry knowledge and advice.

[1] L. Sundqvist Ökvist, A. Dahlstedt, M. Hallin, "The effect on Blast furnace Process of changed Pellet Size as a Result of segregation in Raw Material Handling," Proc. Ironmaking Conference, pp. 167-178, 2001.

[2] M. Blomquist, A. Wernerson, "Range camera on conveyor belts: estimating size distribution and systematic errors due to occlusion," Proc. SPIE - The International Society for Optical Engineering, vol. 3835, pp. 118-126, Sep. 1999.

[3] A Bouajila, M. Bourassa, J.A. Boivin, G. Ouellet, T. I. Martinovic, "On-Line Non-Intrusive Measurement of Greeen Pellet Diameter," Proc. Ironmaking Conference, pp. 1009-1020, 1998.

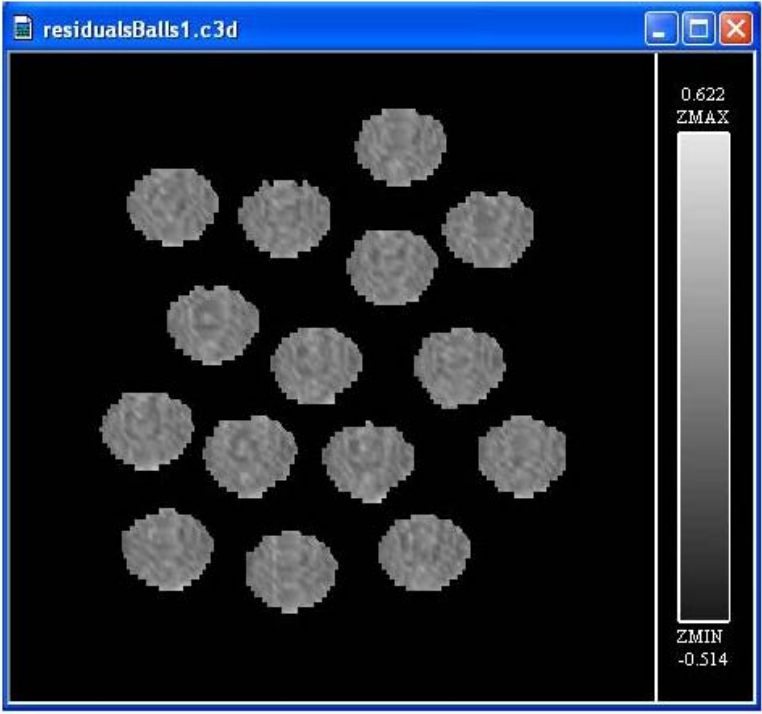

Fig. 6. Sample of residuals for balls with a diameter of $10 \mathrm{~mm}$.

[4] J. E. Larsson, info@mbvsystems.se

[5] "3D scanner: Wikipedia, the free encyclopedia," http://en.wikipedia.org/wiki/3d_scanner.

[6] M. J. Thurley, "On-line 3D Surface Measurement of Iron Ore Green Pellets," Proc. IEEE Conf. Computational Intelligence for Modelling, Control and Automation, Nov 2006.

[7] M.J. Thurley, K.C. Ng, "Identifying, visualizing, and comparing regions in irregularly spaced 3D surface data," Computer Vision and Image Understanding, vol. 98 , no. 2, pp. 239-270, Feb. 2005.

[8] L. Ljung, System identification: theory for the user, Prentice Hall, 1999, 2. ed, ISBN: 0-13-656695-2.

[9] R. Thaka, R. Gentleman, "R: A Language for Data Analysis and Graphics," Journal of Computational and Graphical Statistics, vol. 5, no. 3 , pp. 299-314, 1996, http//www.r-project.org/. 